\title{
Prescripción inadecuada de medicamentos: aportaciones de los paradigmas científicos a su conocimiento'
}

\section{Inadequate drugs prescription: contributions of scientific paradigms to your knowledge}

\author{
Marco Antonio Zavala-González ${ }^{a}$ \\ aniversidad de Guadalajara. Departamento de Salud Pública. \\ Guadalajara, Jalisco, México. \\ E-mail: zgma_51083ळyahoo.com.mx
}

\begin{abstract}
María de los Ángeles Covarrubias-Bermúdez ${ }^{\text {b }}$
bUniversidad de Guadalajara. Departamento de Salud Pública. Guadalajara, Jalisco, México.

E-mail: angelescovarrubiasळhotmail.com
\end{abstract}

\section{Carlos Enrique Cabrera-Pivaral ${ }^{c}$}

'Universidad de Guadalajara. Departamento de Salud Pública. Guadalajara, Jalisco, México.

E-mail: carlos_cabreraœprodigy.net.mx

\section{Igor Martín Ramos-Herrerad}

¿Universidad de Guadalajara. Departamento de Salud Pública. Guadalajara, Jalisco, México.

E-mail: iramosळcucs.udg.mx

\section{Alfredo de Jesús Celis-de-la-Rosa ${ }^{e}$}

eUniversidad de Guadalajara. Departamento de Salud Pública. Guadalajara, Jalisco, México.

E-mail: alfredo_celisळyahoo.com

\section{María de Jesús Orozco-Valerio ${ }^{\dagger}$}

fUniversidad de Guadalajara. Departamento de Salud Pública. Guadalajara, Jalisco, México.

E-mail: maria.orozco.valerioœgmail.com

\section{Correspondencia}

Marco Antonio Zavala-González.

Sierra Mojada, 950, puerta I, edificio n, Planta Alta, Colonia Lomas de Independencia.Guadalajara, Jalisco, México. CEP 44240.

\section{Resumen}

Se realizó una revisión narrativa con análisis temático sobre las aportaciones de los paradigmas científicos al conocimiento de la prescripción inadecuada de medicamentos. Se buscaron artículos de acceso abierto indexados en $\mathrm{PubMed}^{\odot}$ entre 2010-2014, y se sistematizó información sobre el paradigma, tipo de publicación, perspectiva teórica, objetivo, método y resultados. De los 992 artículos encontrados, se seleccionaron 118, y se tomó una muestra propositiva de 15 , según su diseño, representando los cuatro paradigmas. Los artículos positivistas reportaron prevalencia, factores asociados, efectividad de intervenciones y criterios de evaluación; los interpretativos explicaron las causas del problema según los involucrados; los críticos denunciaron la influencia de la industria farmacéutica; y el participativo abordó el problema secundariamente y lo solucionó en un escenario para una enfermedad y grupo farmacológico específicos. Se concluyó que la prescripción inadecuada de medicamentos como problema de investigación en salud pública recibe aportes de los cuatro paradigmas, con dominio del positivismo, lo que se atribuye al carácter paradigmático de la ciencia desde la que se le aborda habitualmente, y que una perspectiva multi-paradigmática es el mejor abordaje.

Palabras clave: Prescripción Inadecuada; Métodos Epidemiológicos; Investigación Cualitativa; Determinantes Sociales de la Salud; Investigación Participativa Basada en la Comunidad; Revisión.

I Trabajo realizado gracias a la Beca Número 329959 para Estudios de Posgrado Nacionales, del Consejo Nacional de Ciencia y Tecnología (CONACYT) de México. 
This study conducted a narrative review with thematic analysis about contributions of scientific paradigms to knowledge of inadequate drugs prescription. We searched open access articles indexed in PubMed $^{\odot}$ between 2010 and 2014, and we systematized information about scientific paradigm, publication type, theoretical perspective, objective, method and results. From the 992 articles found, 118 were selected. From those, we chose a purposive sample of 15 , according to the design of the studies, representing the four paradigms. The positivists articles reported prevalence, associated factors, effectiveness of interventions and evaluation criteria; the interpretive explained the causes of the problem according to those involved; the critics denounced the influence of pharmaceutical industry; and the participative addressed the problem secondarily and solved it in a scenario for a specific disease and pharmacological. We concluded that the inadequate drugs prescription as research problem in public health had contributions from the four paradigms, with dominance of positivism, which is attributed to the paradigmatic perspective of the science, from which it is usually studied, and that a multiparadigmatic perspective is the best approach to the public health issue.

Keywords: Inappropriate Prescribing; Epidemiologic Methods; Qualitative Research; Social Determinants of Health; Community-Based Participatory Research; Review.
En ciencias existe controversia sobre el uso del término "paradigma”, en tanto que Masterman identificó 21 acepciones distintas atribuidas por Kuhn (Nunes, 2014). Por lo que, en correcciones posteriores, Kuhn señaló que un "paradigma científico" debe ser concebido como "un conjunto de métodos, reglas y generalizaciones utilizadas conjuntamente por aquellos entrenados para realizar el trabajo científico de investigación" (Nosnik; Elguea, 1985). Así, el paradigma en que se sitúa el investigador determina las particularidades de su investigación (Ricoy Lorenzo, 2006), las mismas que se reflejarán en las publicaciones que de ella deriven. En este tenor, se reconocen cuatro paradigmas científicos (Heron; Reason, 1997; Hjørland, 2005; Lincoln; Guba, 1994): positivista, interpretativo, crítico y participativo (Cuadro 1).

Así, las aportaciones de cada paradigma científico a un mismo problema de investigación son sustancialmente diferentes entre sí, de modo que, el adecuado conocimiento de un fenómeno debería concatenar las aportaciones de cada uno. En este sentido, Masterman clasifica a las ciencias en cuatro estatus paradigmáticos: no paradigmáticas, paradigmáticas, paradigmáticas duales y paradigmáticas múltiples; lo hace en función de la ausencia de un paradigma dominante, o en función del predominio de uno, dos o más, respectivamente (Marín-Sánchez; Troyano-Rodríguez, 2012).

Por otro lado, al definir la salud pública como "la intervención colectiva, tanto del Estado como de la sociedad civil, orientada a proteger y mejorar la salud de las personas" (OPS, 2002), salta a la vista que los problemas que le atañen son heterogéneos, cambiantes, complejos e íntimamente interrelacionados. Uno de ellos, es la prescripción inadecuada de medicamentos, que consiste en prescribir medicamentos sin atender a las necesidades clínicas de los pacientes, sin tener en cuenta las propiedades cinéticas y dinámicas de los medicamentos, o sin tomar en consideración la posología de los fármacos. La relevancia de este problema radica en su impacto negativo sobre la calidad de vida, la morbilidad y mortalidad poblacional, y sobre la economía personal y estatal (OMS, 1986), dado que su prevalencia llega a superar el 9o\% de las prescripciones, independientemente 
de la enfermedad, grupo farmacoterapéutico y nivel de atención a la salud (Provin et al., 2010; ZavalaGonzález; Lima-Ortiz; Gallegos-Aguilar, 2014; ZavalaGonzález; Sánchez-Santana, 2014).

Por consiguiente, se han propuesto un cúmulo de intervenciones con una efectividad variable, que va desde una reducción de la prevalencia en un 0.2\% (Sellors et al., 2003), hasta un 54.1\% (Altavela;
Jones; Ritter, 2008). Es decir, estos esfuerzos han sido poco fructíferos. En este tenor, se realizó una revisión narrativa de la literatura, con el objetivo de examinar las aportaciones de los paradigmas científicos positivista, interpretativo, crítico y participativo al conocimiento de la prescripción inadecuada de medicamentos como problema de investigación en salud pública.

Cuadro I - Principales características de los paradigmas científicos

\begin{tabular}{|c|c|c|c|c|}
\hline \multirow{2}{*}{ Características } & \multicolumn{4}{|c|}{ Paradigmas científicos } \\
\hline & Positivista & Interpretativo & Crítico & Participativo \\
\hline Objeto & $\begin{array}{l}\text { Atributos de los sujetos y } \\
\text { relaciones entre estos }\end{array}$ & $\begin{array}{l}\text { Perspectiva de los } \\
\text { participantes }\end{array}$ & Denunciar inequidad social & $\begin{array}{l}\text { Problemáticas } \\
\text { comunitarias }\end{array}$ \\
\hline Ontología & $\begin{array}{l}\text { El investigador es árbitro } \\
\text { de la realidad }\end{array}$ & $\begin{array}{l}\text { El investigador es parte } \\
\text { de la realidad }\end{array}$ & $\begin{array}{l}\text { Realismo histórico: crítica a } \\
\text { la realidad }\end{array}$ & $\begin{array}{l}\text { La comunidad modifica } \\
\text { su realidad }\end{array}$ \\
\hline Epistemología & $\begin{array}{l}\text { Objetiva: existe una } \\
\text { realidad más allá de la } \\
\text { subjetividad humana }\end{array}$ & $\begin{array}{l}\text { Subjetiva: la realidad } \\
\text { está dada por } \\
\text { experiencias }\end{array}$ & $\begin{array}{l}\text { Subjetiva: los hallazgos } \\
\text { están dados por valores }\end{array}$ & $\begin{array}{l}\text { Coexistencia de lo } \\
\text { objetivo y lo subjetivo en } \\
\text { la realidad }\end{array}$ \\
\hline Metodología & $\begin{array}{l}\text { Estadística, análisis } \\
\text { estructural-funcionalista }\end{array}$ & $\begin{array}{l}\text { Dialéctica, hermenéutica, } \\
\text { etnográfica }\end{array}$ & $\begin{array}{l}\text { Argumentativa, dialéctica, } \\
\text { dialógica, métodos mixtos }\end{array}$ & $\begin{array}{l}\text { Participación comunitaria } \\
\text { guiada, métodos mixtos }\end{array}$ \\
\hline Axiología & $\begin{array}{l}\text { El investigador deja } \\
\text { de lado sus valores, es } \\
\text { neutral a su objeto }\end{array}$ & $\begin{array}{l}\text { Los valores del } \\
\text { investigador fijan la } \\
\text { teoría y el método }\end{array}$ & $\begin{array}{l}\text { Los valores del investigador } \\
\text { marcan su postura }\end{array}$ & $\begin{array}{l}\text { El investigador se basa } \\
\text { en los valores de la } \\
\text { comunidad }\end{array}$ \\
\hline $\begin{array}{l}\text { Validez y } \\
\text { frabilidad }\end{array}$ & $\begin{array}{l}\text { Rigor metodológico, } \\
\text { certeza estadística, } \\
\text { reproducibilidad }\end{array}$ & $\begin{array}{l}\text { Validación teórica } \\
\text { y por los actores, } \\
\text { "reconstructibilidad" }\end{array}$ & $\begin{array}{l}\text { Generalizaciones por } \\
\text { similitud }\end{array}$ & $\begin{array}{l}\text { Empoderamiento } \\
\text { comunitario, solución de } \\
\text { la problemática }\end{array}$ \\
\hline
\end{tabular}

Fuente: Adaptado de Lincoln y Guba (1994), con información complementaria de Heron y Reason (1997) y Hjørland (2005)

\section{Material y métodos}

Se llevó a cabo una revisión narrativa de la literatura científica en busca de artículos publicados sobre prescripción inadecuada de medicamentos. Se utilizó el método de análisis temático desarrollado por Mays, Pope y Popay (2005), citados por Kastner et al. (2012), para identificar temas prominentes o recurrentes en torno a una gran pregunta de investigación, en este caso, ¿cuáles fueron las aportaciones de los paradigmas científicos positivista, interpretativo, crítico y participativo al conocimiento de la prescripción inadecuada de medicamentos como problema de investigación en salud pública durante el quinquenio 2010-2014?, y resumir los hallazgos de diferentes tipos de estudios publicados.

\section{Estrategia de búsqueda}

Se eligió PubMed ${ }^{\circledR}$ como único sistema de búsqueda de información, pues otorga acceso gratuito al MEDLINE ${ }^{\circledast}$ sin registro ni contraseña, con la posibilidad de elegir entre varias interfaces, enlaces a textos completos de algunos artículos, modalidades de búsqueda avanzadas, enlace a artículos relacionados con una búsqueda previa, y acceso a revistas no médicas (Sobrido Prieto; González Guitian, 2009). La pesquisa de artículos 
se realizó durante octubre y noviembre de 2015 usando términos relacionados con la prescripción inadecuada de medicamentos (Cuadro 2). Estos se buscaron en el título o resumen en inglés de las publicaciones, dada la inviabilidad de su búsqueda en los textos completos, previendo la inclusión de documentos escritos en idiomas distinto al inglés, que serían invisibles con tal protocolo de búsqueda.

Cuadro 2 - Concepto y términos de búsqueda relacionados, utilizados en la revisión de la literatura

\begin{tabular}{|c|c|}
\hline Concepto & Términos de búsqueda \\
\hline $\begin{array}{l}\text { Inadequate } \\
\text { drug } \\
\text { prescription }\end{array}$ & $\begin{array}{l}\text { "Drug utilization review"[MeSH] OR "Medication errors"[MeSH] OR "Medication fails"[Text Word] OR "Prescribing } \\
\text { errors"[Text Word] OR "Prescribing fails"[Text Word] OR "Prescription fails"[Text Word] OR "Prescription quality"[Text Word] } \\
\text { OR "Prescription improvement"[Text Word] OR "Improving prescribing[Text Word] OR "Medication quality"[Text Word] } \\
\text { OR "Drug utilization auditory"[Text Word] OR "Adherence to clinical practice guidelines"[Text Word] OR "Rational drug } \\
\text { utilization"[Text Word] OR "Irrational drug utilization"[Text Word] OR "Inappropriate drug prescription"[Text Word] OR } \\
\text { "Inappropriate drug utilization"[Text Word] OR "Adequate utilization of therapeutic resources"[Text Word] OR "Therapeutic } \\
\text { resources utilization"[Text Word] OR "Pharmacological treatment quality"[Text Word] }\end{array}$ \\
\hline
\end{tabular}

Nota: El concepto y los términos de búsqueda se presentan en idioma inglés, puesto que es el idioma con el que se indexan

\section{Selección de referencias}

Se seleccionaron artículos científicos publicados durante el periodo de estudio de acceso gratuito al texto completo (por limitaciones de recursos económicos), escritos en inglés, español o portugués (idiomas comprendidos por los autores), provenientes de cualquier país. Se consideraron como artículos científicos: artículos originales, revisiones, textos especiales, artículos de reflexión, debates, ensayos y opiniones. Estos, según los criterios de Day (2005), usados anteriormente en investigaciones sobre bases teóricas de la producción científica (Cabrera Arana, 2005, 2007), al considerar que dichas publicaciones presentan nuevos conocimientos o métodos y discusiones del saber al exponer nuevos enfoques o hipótesis.

Se encontraron 992 publicaciones, a las que se aplicaron tres rondas de selección (Figura 1). La primera, al satisfacer los criterios definidos en el párrafo anterior, resultó en la exclusión 653 publicaciones por acceso no gratuito, 7 por idioma, 1 por duplicada, y 6 por incumplir criterios de Day, quedando 325 artículos para la siguiente ronda. La segunda ronda consistió en la lectura de los resúmenes (cuando estaban disponibles) de las publicaciones obtenidas en la primera ronda, con lo que se excluyeron los artículos en los que no se trabajó con el concepto de prescripción inadecuada de medicamentos. En el caso de los textos cuya estructura no incluye resumen, como ensayos $\mathrm{u}$ opiniones, se leyó el texto completo y se aplicó el mismo criterio. Así, se eliminaron 207 publicaciones: 39 sobre efectos clínicos de medicamentos, 33 sobre patrones de uso y costo de medicamentos, 31 sobre evolución clínica de las enfermedades, 25 sobre desarrollo y mejora de formulaciones, 16 sobre adherencia al tratamiento, 12 sobre manejo gerencial y calidad de las formulaciones, 7 sobre prácticas médicas, 6 sobre auditoría hospitalaria, 6 sobre políticas públicas, y 4 que abordaron otros temas una sola vez, más 28 artículos a los que no se logró tener acceso a la revista. Como resultado, se identificaron 118 artículos útiles, que abordaron concretamente el tema de esta revisión.

En la tercera ronda, se realizó un muestreo propositivo en el que se releyeron los textos resultantes de la ronda de selección anterior y se clasificaron según su paradigma científico. Se encontraron: 109 positivistas, 5 interpretativos, 3 críticos y 1 participativo. Se incluyeron todos los interpretativos, críticos y participativos, mientras que, en el caso de los positivistas, dado su desproporcionado predominio, con fines ilustrativos, se llevó a cabo una selección propositiva, eligiendo aleatoriamente un artículo por cada diseño descrito por Hernández-Ávila, GarridoLatorre y López-Moreno (200o), más un estudio de construcción y validación de escalas según lo descrito por Luján-Tangarife y Cardona-Arias (2015). Así, se incluyeron 6 artículos pertenecientes al paradigma positivista. 
Figura I - Proceso de selección de artículos

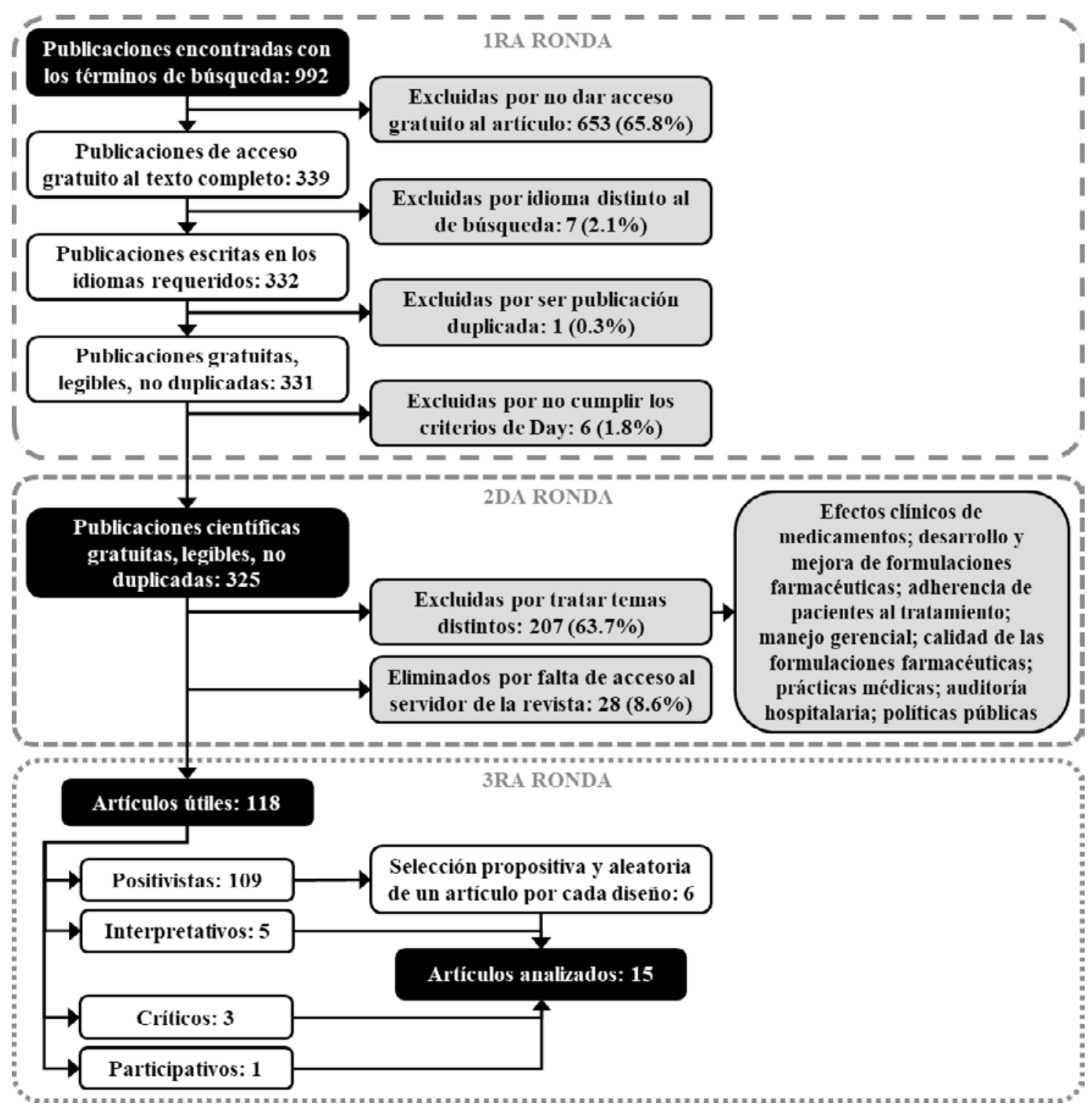

\section{Extracción y análisis de datos}

Los datos se extrajeron en una matriz (Cuadros 3 y 4) donde se incluyó el primer autor, el año y el país de publicación, el paradigma científico, el tipo de publicación, la perspectiva o modelo teórico, el objetivo, el método y los resultados de la investigación. El paradigma científico fue identificado por los autores según las características descritas en el Cuadro 1, cuando no se refirió textualmente. El tipo de publicación fue el referido en el manuscrito según los criterios de la revista correspondiente. La perspectiva o modelo teórico fue el referido al interior del manuscrito, salvo en los positivistas, donde se asumió que todos se encontraban dentro del pospositivismo por ser la perspectiva vigente. El objetivo fue tomado textualmente, al igual que el método, mientras que, en cuanto a resultados, se tomaron solo los específicos al objeto de la revisión. Este análisis se realizó entre pares buscando consenso, sumando un tercero en caso de discordancia. 


\begin{tabular}{|c|c|c|c|c|c|}
\hline 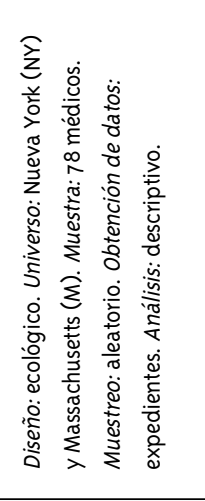 & & 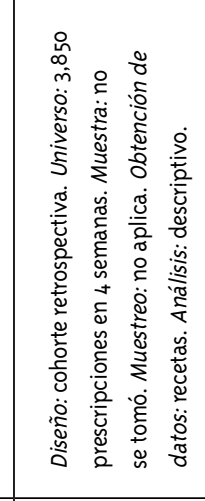 & & 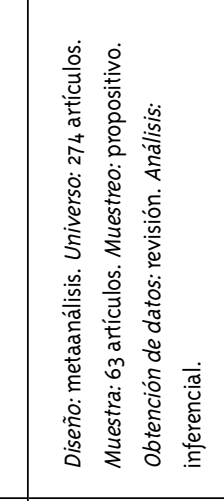 & \\
\hline & & & & & \\
\hline$\sqrt{1}$ & & $\frac{9}{1}$ & 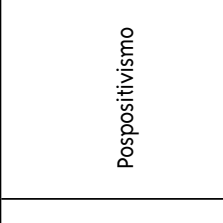 & $\begin{array}{l}\frac{9}{1} \\
\underline{1} \\
\end{array}$ & 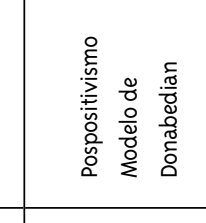 \\
\hline 重蓑 & 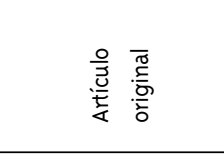 & $\frac{\sqrt[n]{1}}{12}$ & 管高 & 景高 & 裳畄 \\
\hline$\frac{\underline{n}}{1}$ & $\frac{\sqrt[n]{1}}{1}$ & $\frac{\sqrt{1}}{1}$ & $\frac{\sqrt[n]{1}}{1}$ & $\frac{n}{1}$ & $\sqrt{1}$ \\
\hline $\begin{array}{l}1 \\
\end{array}$ & 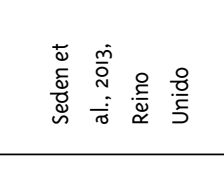 & 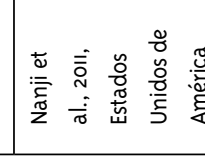 & 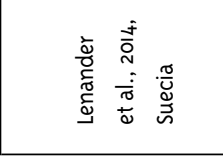 & 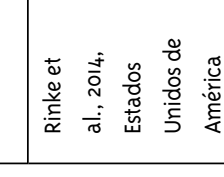 & 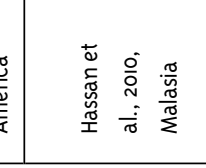 \\
\hline
\end{tabular}




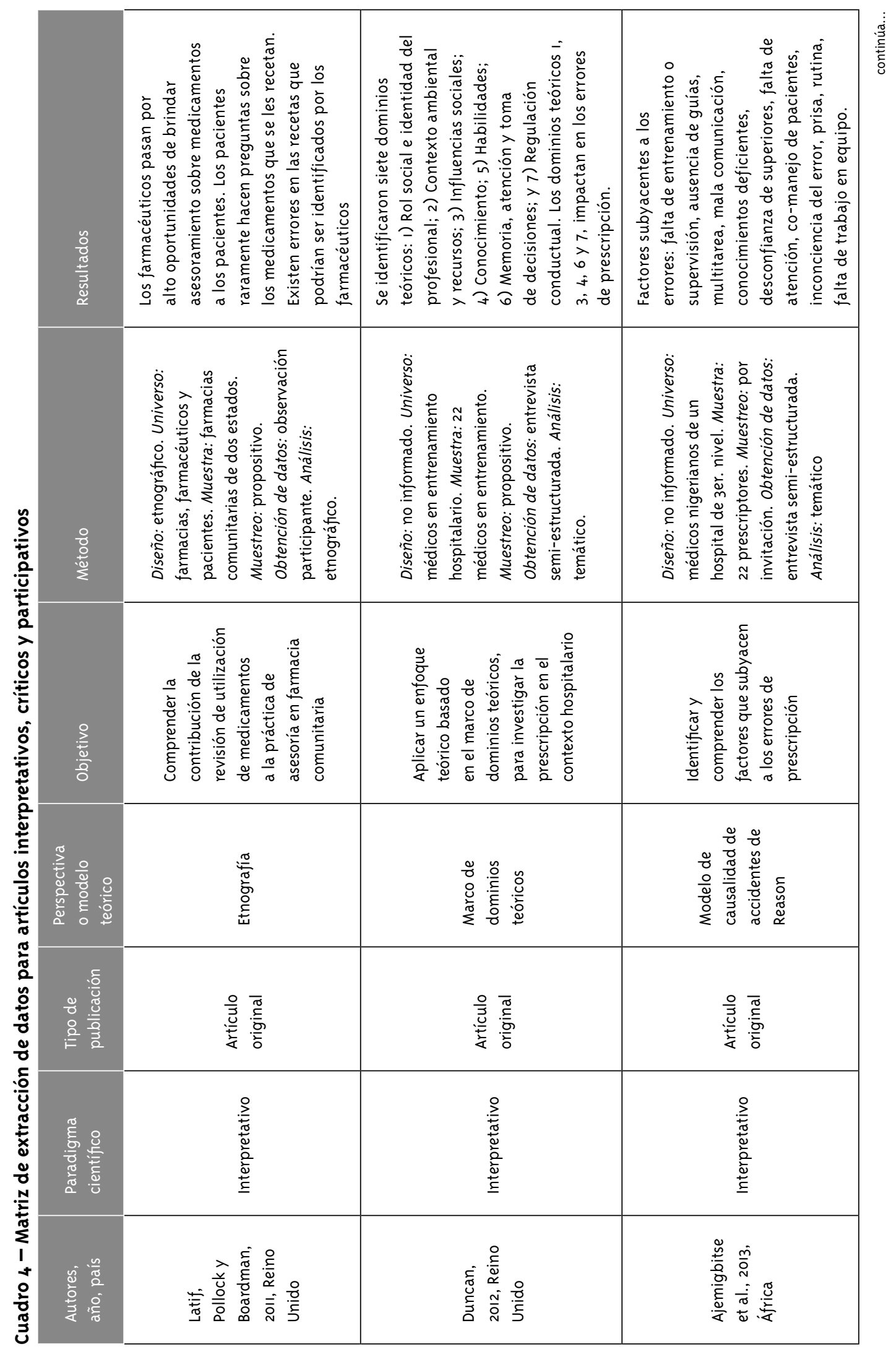




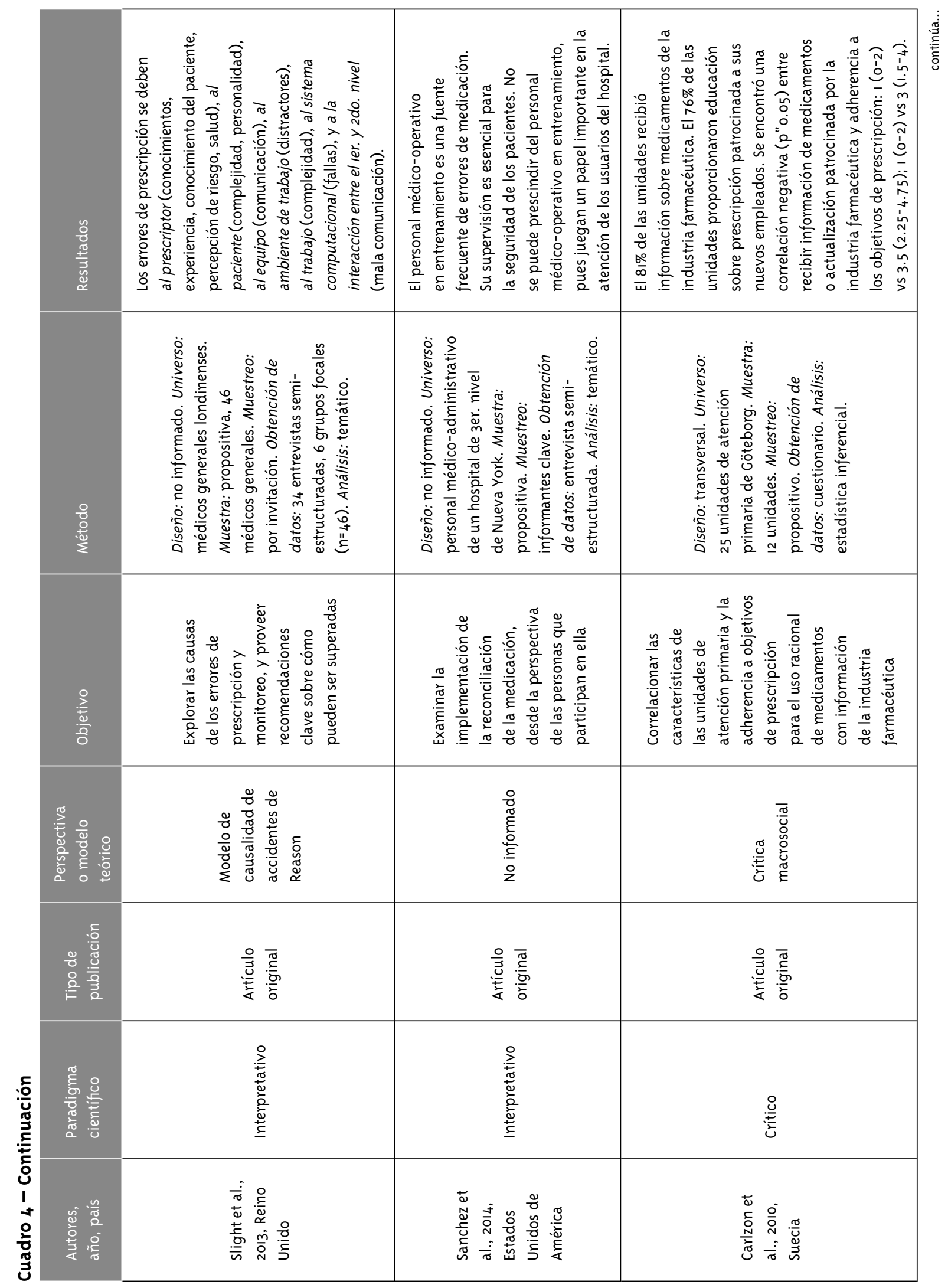




\begin{tabular}{|c|c|c|c|}
\hline 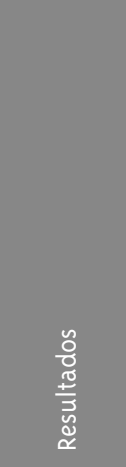 & 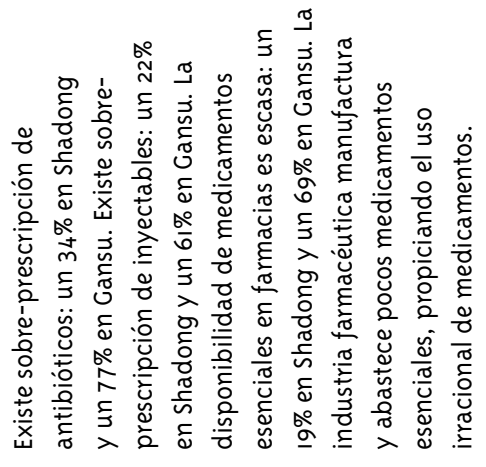 & 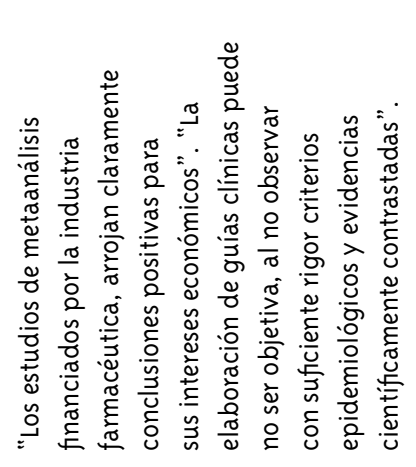 & 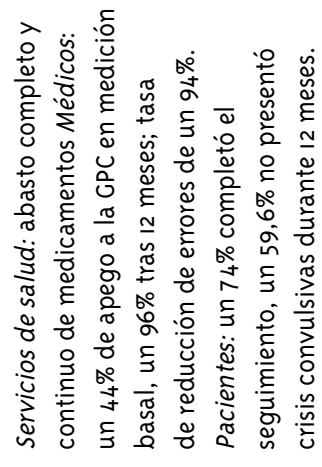 \\
\hline $\begin{array}{l}\frac{0}{0} \\
\frac{0}{0} \\
\frac{.0}{2}\end{array}$ & 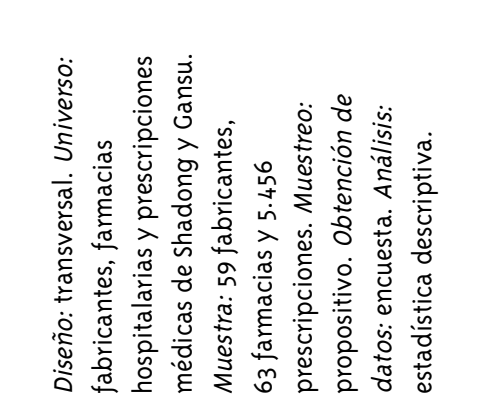 & 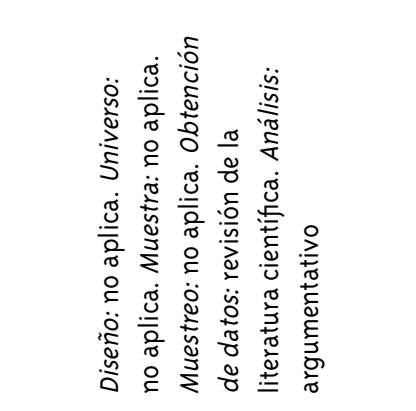 & 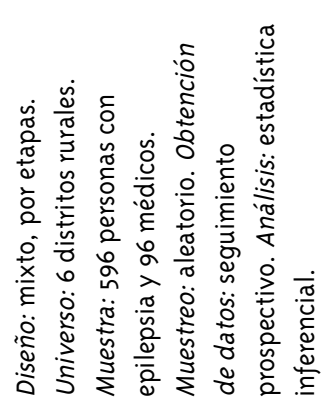 \\
\hline 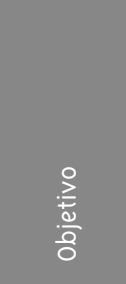 & 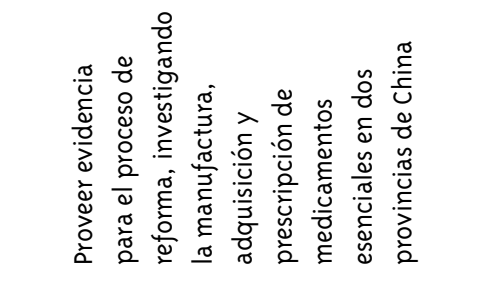 & 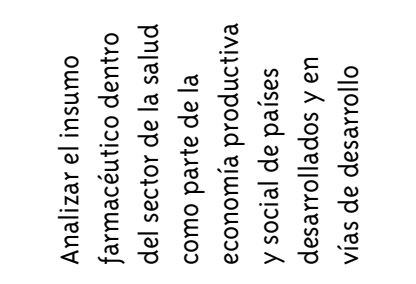 & 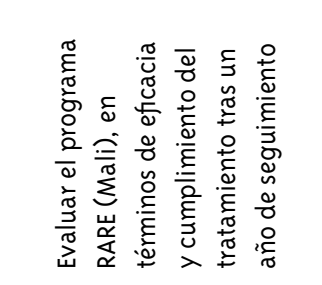 \\
\hline 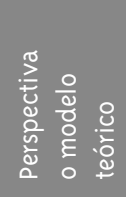 & 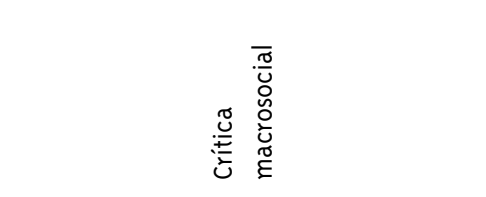 & 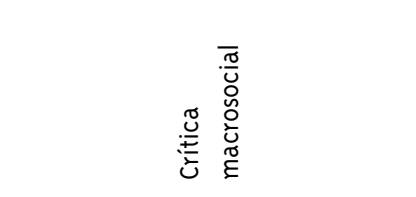 & 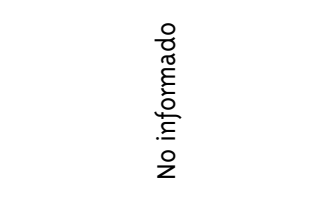 \\
\hline 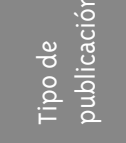 & 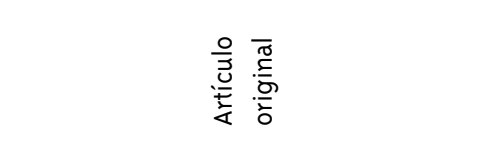 & 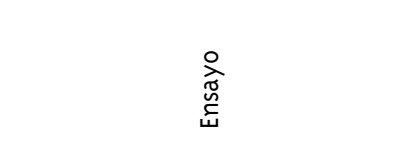 & 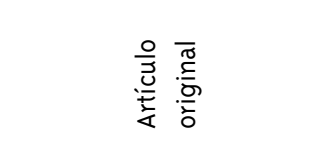 \\
\hline 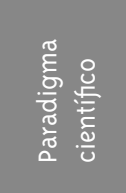 & 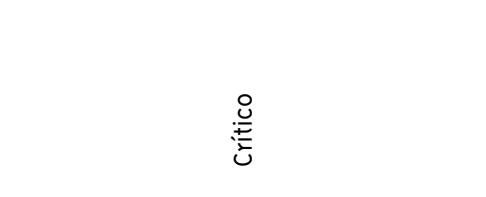 & 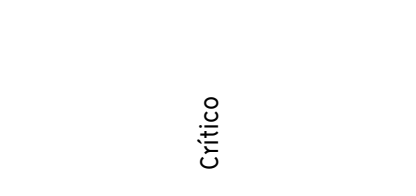 & 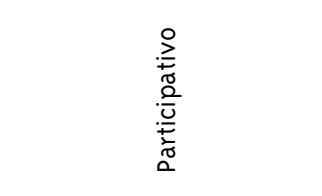 \\
\hline 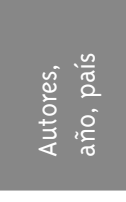 & 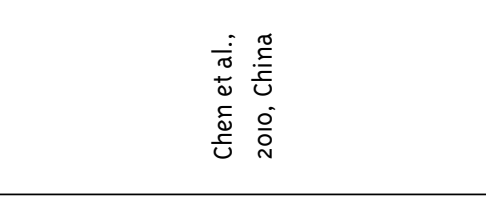 & 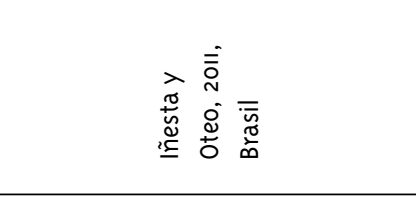 & 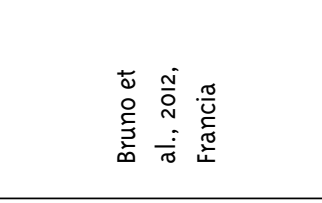 \\
\hline
\end{tabular}


Se obtuvieron distribuciones de frecuencias para describir las características bibliométricas de los artículos incluidos, mientras que sus contenidos fueron analizados en cuatro ejes temáticos (Mays; Pope; Popay, 2005), uno por cada paradigma, puntualizando las aportaciones de cada uno.

\section{Resultados}

Se analizaron 15 artículos que representaron los cuatro paradigmas científicos: seis (40\%) del positivista, cinco (33\%) del interpretativo, tres (20\%) del crítico y uno (7\%) del participativo. Según el lugar de origen de las investigaciones, cuatro (27\%) fueron de Estados Unidos de América (EE. UU.), cuatro (27\%) de Reino Unido, dos (13\%) de Suecia y los cinco restantes de África, Brasil, China, Francia y Malasia, cada uno con el $7 \%$ del total. Las principales características de los artículos analizados por paradigma se presentan en los Cuadros 3 y 4.

\section{Aportaciones del paradigma positivista}

Los resultados de estos trabajos evidencian una proporción variable de prescripciones inadecuadas de medicamentos (Abramson et al., 2012; Nanji et al., 2011; Seden et al., 2013), que van desde el 1,7\% (Seden et al., 2013) hasta el 43,8\% (Abramson et al., 2012) a nivel comunitario e individual en diferentes escenarios, mediante evaluación transversal (Abramson et al., 2012; Seden et al., 2013) o longitudinal (Nanji et al., 2011). Además, reportan intervenciones educativas, gerenciales e informáticas (Lenander et al., 2014; Rinke et al., 2014), de efectividad variable, con tasas de reducción de errores del 1,73\% (Lenander et al., 2014) al 87\% (Rinke et al., 2014), que, dada la heterogeneidad de criterios de medición, no siempre pueden concatenarse para realizar un metaanálisis (Rinke et al., 2014) que permita tomar decisiones gerenciales desde este paradigma. Asimismo, se encontró que se desarrollan nuevos instrumentos para determinar, con mejor precisión, los errores de prescripción (Hassan et al., 2010), lo que implica cambios frecuentes en la definición del problema que favorecen la heterogeneidad de resultados en cuanto a la prevalencia y la incidencia, y dificulta los metaanálisis (Rinke et al., 2014).

\section{Aportaciones del paradigma interpretativo}

Los artículos interpretativos trabajan desde perspectivas teóricas variadas, donde el modelo de causalidad de accidentes de Reason es la más frecuente (Ajemigbitse et al., 2013; Slight et al., 2013). Estos trabajos aportan la visión de los actores que participan en la prescripción inadecuada de medicamentos, principalmente del personal médico (Ajemigbitse et al., 2013; Duncan et al., 2012; Sanchez et al., 2014; Slight et al., 2013) y farmacéutico (Latif; Pollock; Boardman, 2011), aunque otros profesionales de la salud también están involucrados en este problema.

Los resultados de estos estudios evidencian situaciones que participan en la génesis del error médico, como distractores ambientales (Ajemigbitse et al., 2013; Duncan et al., 2012; Slight et al., 2013), presiones laborales (Ajemigbitse et al., 2013; Duncan et al., 2012; Slight et al., 2013), arquitectura de software deficiente (Slight et al., 2013), falta de supervisión (Ajemigbitse et al., 2013; Latif; Pollock; Boardman, 2011; Sanchez et al., 2014) y retroalimentación (Ajemigbitse et al., 2013; Slight et al., 2013), ausencia de guías de práctica clínica en la unidad médica (Ajemigbitse et al., 2013), co-manejo de pacientes (Ajemigbitse et al., 2013), falta de colaboración entre pares (Ajemigbitse et al., 2013; Slight et al., 2013) y otros profesionales de la salud (Ajemigbitse et al., 2013), entre otros factores que explican la variabilidad en la efectividad de las intervenciones realizadas desde el positivismo (Lenander et al., 2014; Rinke et al., 2014) para solucionar el problema, que desde su concepción no inciden sobre las condiciones informadas en el paradigma interpretativo por los protagonistas del problema.

\section{Aportaciones del paradigma crítico}

Los artículos críticos hacen una crítica macrosocial, enfocándose en elementos estructurales sociales y de tenencia de medios de producción (Heron; Reason, 1997; Hjørland, 2005; Lincoln; Guba, 1994). En este sentido, empleando métodos cuantitativos (Carlzon et al., 2010; Chen et al., 2010) y argumentativos (Iñesta; Oteo, 2011), denuncian la influencia de intereses 
financieros de la industria farmacéutica sobre el uso inadecuado de medicamentos (Carlzon et al., 2010; Chen et al., 2010; Iñesta; Oteo, 2011), que permean en el contexto tanto de la medicina privada como de la subsidiada por el Estado (Carlzon et al., 2010; Chen et al., 2010; Iñesta; Oteo, 2011), de la medicina basada en evidencias (Iñesta; Oteo, 2011), de la formación de médicos en pre- y posgrado (Carlzon et al., 2010), y de la disponibilidad de medicamentos esenciales a nivel institucional, estatal y nacional (Chen et al., 2010). Según los estudios incluidos en esta revisión, esto es invisible para los paradigmas positivista e interpretativo.

\section{Aportación del paradigma participativo}

Los artículos desde este paradigma son escasos, pues solo se encontró uno (Bruno et al., 2012). Este abordó la prescripción inadecuada de medicamentos secundariamente, ya que no fue el foco de interés de la comunidad, sino el manejo de una enfermedad per se. En este sentido, el estudio identificado sugiere la superioridad de este paradigma en la solución del problema, puesto que logró una tasa de reducción de errores de un 94\% (Bruno et al., 2012), a la que no se acerca ninguna intervención positivista (Lenander et al., 2014; Rinke et al., 2014), lo que podría atribuirse a que desde el participativo se consideraron los aportes positivistas, interpretativos y críticos, e incidieron sobre múltiples aristas del problema. Empero, esta comparación se basa en un único estudio participativo frente a varios positivistas, por lo que la superioridad sugerida queda en calidad de hipótesis que habrá de comprobarse posteriormente, cuando se cuente con suficientes investigaciones participativas publicadas sobre el tema.

\section{Discusión}

Este es el primer trabajo conocido por los autores, en el que se examinan las aportaciones de los paradigmas científicos al conocimiento de la prescripción inadecuada de medicamentos como problema de investigación. En este sentido, los resultados evidencian que la producción científica en torno a este está dominada por el paradigma positivista, ya que la ciencia desde la que se aborda principalmente, es la farmacoepidemiología, encargada de estudiar "la utilización de los medicamentos por la población una vez que son comercializados" (Strom, 2006). Si bien se valen de métodos de investigación propios, denominados "estudios de utilización de medicamentos", estos se fundamentan en los principios teórico-metodológicos de la epidemiología (Strom, 20o6), lo que le otorga a esta ciencia un carácter paradigmático según la taxonomía propuesta por Masterman (MarínSánchez; Troyano-Rodríguez, 2012).

No obstante, este predominio del paradigma positivista ha sido observado en la producción científica publicada en revistas latinoamericanas de salud pública (Cabrera Arana, 2005, 2007) en diferentes ciencias de la salud, como epidemiología (Saldarriaga, 2013), enfermería (Triviño; Sanhueza, 2005) y medicina (Coello Valdés; Blanco Balbeíto; Reyes Orama, 2012), y en la propia salud pública como campo disciplinar (Nunes, 2014). Esto puede atribuirse a la vigencia y predominio de los modelos "biomédico", "epidemiológico clásico" e "higienista preventivo" en la salud pública contemporánea por sobre el modelo "socio-médico", lo que invisibiliza factores psíquicos, sociales y culturales, que, junto a los biológicos, determinan el proceso salud-enfermedad (HernándezGirón; Orozco-Núñez; Arredondo-López, 2012). Por consiguiente, se puede afirmar que las ciencias de la salud pública son paradigmáticas, y que el paradigma dominante en la mayoría de sus ramificaciones es el positivismo, de modo que los resultados de esta revisión sólo reafirman este hecho.

Sin embargo, en el caso del problema abordado en este trabajo, es posible que exista una producción más amplia de estudios sobre prescripción inadecuada de medicamentos, realizados desde los paradigmas interpretativo, crítico y participativo, publicados en revistas no indexadas en PubMed(C), o indexadas ahí pero inaccesibles para los autores, o bien ubicada en tesis no publicadas correspondientes a la "literatura gris” (Rumsey, 2008), que no se incluyeron en esta revisión, lo que muestra su limitación por seleccionar a priori manuscritos de la mejor calidad (Rumsey, 2008). Además, posiblemente se excluyeron trabajos que abordan el tema secundariamente desde el paradigma interpretativo, crítico o participativo, pero que en sus títulos y resúmenes no incluyeron los 
términos de esta revisión (Cuadro 2), al usar formas inusuales de referirse al problema regionalmente (Rumsey, 2008). Esto es un potencial sesgo de selección, que se puede atribuir a la búsqueda en inglés, idioma de indexación en bases de datos internacionalmente (Rumsey, 2008; Sobrido Prieto; González Guitian, 2009).

Tomando en cuenta lo anterior, los resultados de esta revisión demuestran un claro predominio del positivismo en la producción científica sobre prescripción inadecuada de medicamentos. En este sentido, tales estudios solo evidencian una y otra vez el problema en diversos contextos (Abramson et al., 2012; Nanji et al., 2011; Seden et al., 2013) y difunden resultados de intervenciones que, si bien han dado resultados estadísticamente significativos (Lenander et al., 2014; Rinke et al. 2014), no han logrado abatir el problema o reducirlo hasta un mínimo aceptable, lo que, secundariamente, ha sido logrado desde el paradigma participativo (Bruno et al., 2012). Por lo tanto, esta revisión demuestra lo referido por Coello Valdés, Blanco Balbeíto y Reyes Orama (2012), quienes, aludiendo a las ciencias médicas, señalaron que:

la unilateralidad en el uso de cualquiera de los paradigmas limita la profundidad del análisis y el alcance de los resultados, y que la interacción de estos garantiza niveles cada vez mayores de objetividad y profundidad del saber, de modo que un abordaje multi-paradigmático contribuye a un mejor desarrollo y calidad de las investigaciones realizadas. (p. 145)

Lo que parece lograrse al investigar desde el paradigma participativo es que el estudio combina los aportes de los paradigmas positivista, interpretativo y crítico (Heron; Reason, 1997; Hjørland, 2005; Lincoln; Guba, 1994).

Sin embargo, si bien las investigaciones participativas son principalmente intervenciones comunitarias (Heron; Reason, 1997; Hjørland, 2005; Lincoln; Guba, 1994), estas son escasas respecto a la producción científica desde otros paradigmas, y se suelen centrar en problemas relacionados con la promoción de la salud (Coello Valdés; Blanco Balbeíto; Reyes Orama, 2012; George et al., 2015; Saldarriaga, 2013; Triviño; Sanhueza, 2005). Así, sus objetos de estudio son definidos por las comunidades (Heron; Reason, 1997; Hjørland, 2005; Lincoln; Guba, 1994), lo que pone en desventaja problemas relevantes para la salud pública global, como la prescripción inadecuada de medicamentos, frente a otros problemas de carácter fuertemente local, y, como resultado, los problemas globales se quedan sin atención indefinidamente, hasta que se crea consciencia de ellos.

Se puede concluir entonces que la prescripción inadecuada de medicamentos como problema de investigación recibe aportes de los cuatro paradigmas científicos, con claro dominio del positivismo. Esto se atribuye al carácter paradigmático de la ciencia desde la que se le aborda habitualmente, y esta unilateralidad en torno al problema es perjudicial para su solución, pues limita la profundidad y alcance de las investigaciones. Mientras tanto, cuando se integran elementos de otros paradigmas, como sucede en el participativo, se obtienen mejores resultados, sin embargo, las comunidades no abordan problemas como el tratado en esta revisión, pues son invisibles para población lega. Por esto, los investigadores deberían optar por realizar investigaciones multi-paradigmáticas de interés global, nacional o regional, al margen de los intereses locales de las comunidades, por los que vela el paradigma participativo.

\section{Referencias}

ABRAMSON, E. L. et al. Ambulatory prescribing errors among community-based providers in two states. Journal of the American Medical Informatics Association, Philadelphia, v. 19, n. 4, p. 644-648, 2012. Disponível em: <http://bit. ly/2kAFWT3>. Acesso em: 15 fev. 2017.

AJEMIGBITSE, A. A. et al. A qualitative study of causes of prescribing errors among junior medical doctors in a Nigeria in-patient setting. Annals of African Medicine, Sokoto, v. 12, n. 4, p. 223-231, 2013. Disponível em: <http://bit.ly/2lOQp2t>. Acesso em: 15 fev. 2017.

ALTAVELA, J. L.; JONES, M. K.; RITTER, M. A prospective trial of a clinical pharmacy intervention in a primary care practice in a 
capitated payment system. Journal of Managed Care and Specialty Pharmacy, Alexandria, v. 14, n. 9, p. 831-843, 2008. Disponível em: <http://bit. ly/2lgMlql>. Acesso em: 15 fev. 2017.

BRUNO, E. et al. Results of an action-research on epilepsy in rural Mali. PLoS One, San Francisco, v. 7, n. 8, p. 1-7, 2012. Disponível em: <http://bit. ly/2lnb1xF>. Acesso em: 15 feb. 2017.

CABRERA ARANA, G. A. Teorías y modelos en la Revista Facultad Nacional de Salud Pública de la Universidad de Antioquia, 2000-2004. Revista Facultad Nacional de Salud Pública, Medellín, v. 23, n. 2, p. 78-82, 2005. Disponível em: <http:// bit.ly/2kV6qlJ>. Acesso em: 15 fev. 2017.

CABRERA ARANA, G. A. Uso de teorías y modelos en artículos de una revista latinoamericana de salud pública, 200o-2004. Revista de Saúde Pública, São Paulo, v. 41, n. 6, p. 963-969, 2007. Disponível em: <http://bit.ly/2kV1XPT>. Acesso em: 15 fev. 2017.

CARLZON, D. et al. Characteristics of primary health care units with focus on drug information from pharmaceutical industry and adherence to prescribing objectives: a cross-sectional study. BMC Clinical Pharmacology, London, v. 1o, n. 4, p. 1-5, 2010. Disponível em: <http://bit.ly/2lnjktx>. Acesso em: 15 fev. 2017.

CHEN, W. et al. Availability and use of essential medicines in China: manufacturing, supply, and prescribing in Shandong and Gansu provinces. BMC Health Services Research, London, v. 10, n. 211, p. 1-8, 2010. Disponível em: <https://bit. ly/2AmrzPk>. Acesso em: 15 fev. 2017.

COELLO VALDÉS, E.; BLANCO BALBEÍTO, N.; REYES ORAMA, Y. Los paradigmas cuantitativos y cualitativos en el conocimiento de las ciencias médicas con enfoque filosófico-epistemológico. Edumecentro, Santa Clara, v. 4, n. 2, p. 137-146, 2012. Disponível em: <http://bit.ly/2lnBY4L〉. Acesso em: 15 fev. 2017.

DAY, R. A. Cómo escribir y publicar trabajos científicos. 3. ed. Washington, DC: Organización Panamericana de la Salud, 2005. Disponível em: <http://bit.ly/19XuMlL>. Acesso em: 15 fev. 2017.
DUNCAN, E. M. et al. Learning curves, taking instructions, and patient safety: using a theoretical domains framework in an interview study to investigate prescribing errors among trainee doctors. Implementation Science, London, v. 7, p. 86, 2012. Disponível em: <http://bit. ly/2lOQ3sF>. Acesso em: 15 fev. 2017.

GEORGE, A. S. et al. Community participation in health systems research: a systematic review assessing the state of research, the nature of interventions involved and the features of engagement with communities. PLoS One, San Francisco, v. 10, n. 10, p. 1-25, 2015. Disponível em: <http://bit.ly/2kSnCWL . Acesso em: 15 fev. 2017.

HASSAN, N. B. et al. Development and validation of a new prescription quality index. British Journal of Clinical Pharmacology, London, v. 7o, n. 4, p. 500-513, 2010. Disponível em: <http://bit. ly/2lS3THo>. Acesso em: 15 fev. 2017.

HERNÁNDEZ-ÁVILA, M.; GARRIDO-LATORRE, F.; LÓPEZ-MORENO, S. Diseño de estudios epidemiológicos. Salud Pública de México, Cuernavaca, v. 42, n. 2, p. 144-154, 2000.

Disponível em: <http://bit.ly/1PogY4C $>$. Acesso em: 15 fev. 2017.

HERNÁNDEZ-GIRÓN, C.; OROZCO-NÚÑEZ, E.; ARREDONDO-LÓPEZ, A. Modelos conceptuales y paradigmas en salud pública. Revista de Salud Pública, Bogotá, DC, v. 14, n. 2, p. 315-324, 2012. Disponível em: <http://bit.ly/2lR1a4g>. Acesso em: 15 fev. 2017.

HERON, J.; REASON, P. A participatory inquiry paradigm. Qualitative Inquiry, Thousand Oaks, v. 3, n. 3, p. 274-294, 1997. Disponível em: <http:// bit.ly/2lQW84A>. Acesso em: 15 fev. 2017.

HJøRLAND, B. Empiricism, rationalism and positivism in library and information science. Journal of Documentation, London, v. 61, n. 1, p. 130-155, 2005. Disponível em: <http://bit. ly/2krvIcD>. Acesso em: 15 fev. 2017.

IÑESTA, A.; OTEO, L. A. La industria farmacéutica y la sostenibilidad de los sistemas de salud en países desarrollados y América Latina. Ciência e Saúde Coletiva, Rio de Janeiro, v. 16, n. 6, p. 2713- 
2724, 2011. Disponível em: <http://bit.ly/2lOU7Jz>. Acesso em: 15 fev. 2017.

KASTNER, M. et al. What is the most appropriate knowledge synthesis method to conduct a review? Protocol for a scoping review. $B M C$ Medical Research Methodology, London, v. 12, n. 114, p. 1-10, 2012. Disponível em: <https://bit. ly/2LqbgGq >. Acesso em: 15 fev. 2017.

LATIF, A.; POLLOCK, K.; BOARDMAN, H. F. The contribution of the Medicines Use Review (MUR) consultation to counseling practice in community pharmacies. Patient Education and Counseling, Princeton, v. 83, n. 3, p. 336-344, 2011. Disponível em: <http://bit.ly/2kNkDPd>. Acesso em: 15 fev. 2017.

LENANDER, C. et al. Effects of a pharmacist-led structured medication review in primary care on drug-related problems and hospital admission rates: a randomized controlled trial. Scandinavian Journal of Primary Health Care, Stockholm, v. 32, n. 4, p. 18o-186, 2014. Disponível em: <http://bit. ly/2lVJKQa>. Acesso em: 15 fev. 2017.

LINCOLN, Y. S.; GUBA, E. G. Paradigmatic controversies, contradictions and emerging cofluences. In: DENZIN, N. K.; LINCOLN, Y. S.

(Ed.). Handbook of qualitative research. Thousand Oaks: Sage Publications, 1994. p. 163-168.

Disponível em: <http://bit.ly/2kUkmwb>. Acesso em: 15 fev. 2017.

LUJÁN-TANGARIFE, J. A.; CARDONA-ARIAS, J. A. Construcción y validación de escalas de medición en salud: revisión de propiedades psicométricas. Archivos de Medicina, Oviedo, v. 11, n. 3, p. 1-10, 2015. Disponível em: <http://bit.ly/2bgYwLO>. Acesso em: 15 fev. 2017.

MARÍN-SÁNCHEZ, M.; TROYANO-RODRÍGUEZ, Y. Antecedentes históricos, concepto, enfoques y objeto de estudio en la psicología social. In: MARÍN-SÁNCHEZ, M.; MARTÍNEZ-PECINO, R. (Coord.). Introducción a la psicología social. Madrid: Pirámide, 2012. p. 22-23.

MAYS, N.; POPE, C.; POPAY, J. Systematically reviewing qualitative and quantitative evidence to inform management and policy-making in the health field. Journal of Health Services Research and Policy, Edinburgh, v. 10, n. 1, p. 6-20, 2005. Disponível em: <http://bit.ly/2lT6LD8>. Acesso em: 15 fev. 2017.

NANJI, K. C. et al. Errors associated with outpatient computerized prescribing systems. Journal of the American Medical Informatics Association, Philadelphia, v. 18, n. 6, p. 767-773, 2011. Disponível em: <http://bit.ly/2kBNipD>. Acesso em: 15 fev. 2017.

NOSNIK, A.; ELGUEA, J. La discusión sobre el crecimiento del conocimiento científico en el cuento de la filosofía de la ciencia. Estudios, México, DF, n. 2, 1985. Disponível em: <https://bit. ly/2NSxQob>. Acesso em: 15 fev. 2017.

NUNES, E. D. Paradigmas de la salud colectiva: breve reflexión. Salud Colectiva, Buenos Aires, v. 10, n. 1, p. 57-65, 2014. Disponível em: <http:// bit.ly/2kL5YnL>. Acesso em: 15 fev. 2017.

OMS - ORGANIZACIÓN MUNDIAL DE LA SALUD. Uso racional de los medicamentos: informe de la Conferencia de Expertos, Nairobi, 25-29 de noviembre de 1985. Ginebra, 1986. Disponível em: <http://bit.ly/1Qzq5X3>. Acesso em: 15 fev. 2017.

OPS - ORGANIZACIÓN PANAMERICANA DE LA

SALUD. La salud pública en las Américas: nuevos conceptos, análisis del desempeño y bases para la acción. Washington, DC, 2002. Disponível em: <http://bit.ly/2kLtBfX>. Acesso em: 15 fev. 2017.

PROVIN, M. et al. Atenção farmacêutica em Goiânia: inserção do farmacêutico na Estratégia Saúde da Família. Saúde e Sociedade, São Paulo, v. 19, n. 3, p. 717-723, 2010. Disponível em: <http:// bit.ly/2e1SO1r>. Acesso em: 15 fev. 2017.

RICOY LORENZO, C. Contribución sobre los paradigmas de investigación. Educação, Santa María, v. 31, n. 1, p. 11-22, 2006. Disponível em: <http://bit.ly/2ljepKu>. Acesso em: 15 fev. 2017.

RINKE, M. L. et al. Interventions to reduce pediatric medication errors: a systematic review. Pediatrics, Springfield, v. 134, n. 2, p. 338-36o, 2014. Disponível em: <http://bit.ly/2lSguNX〉. Acesso em: 15 fev. 2017.

RUMSEY, S. How to find information: a guide for researchers. 2. ed. London: McGraw-Hill: Open 
University Press, 2008. Disponível em: <http://bit. ly/2lfpfyz>. Acesso em: 15 fev. 2017.

SALDARRIAGA, E. A. Integración de métodos cuantitativos y cualitativos en epidemiología: una necesidad imperante. CES Salud Pública, Medellín, v. 4, n. 1, p. 6o-64, 2013. Disponível em: <http://bit.ly/2lQSLOm>. Acesso em: 15 fev. 2017.

SANCHEZ, S. H. et al. Implementing medication reconciliation from the planner's perspective: a qualitative study. BMC Health Services Research, London, v. 14, n. 290, p. 1-10, 2014. Disponível em: <http://bit.ly/2kW922B>. Acesso em: 15 fev. 2017.

SEDEN, K. et al. Cross-sectional study of prescribing errors in patients admitted to nine hospitals across North West England. BMJ Open, London, v. 3, p. 1-14, 2013. Disponível em: <http:// bit.ly/2kUVi8E >. Acesso em: 15 fev. 2017.

SELLORS, J. et al. A randomized controlled trial of a pharmacist consultation program for family physicians and their elderly patients. Canadian Medical Association Journal, Ottawa, v. 169, n. 1, p. 17-22, 2003. Disponível em: <http://bit. ly/2kURRig>. Acesso em: 15 fev. 2017.

SLIGHT, S. P. et al. The causes of prescribing errors in English general practices: a qualitative study. British Journal of General Practice, London, v. 63, n. 615, p. e713-e720, 2013. Disponível em: <http://bit.ly/2lODw8r >. Acesso em: 15 fev. 2017.
SOBRIDO PRIETO, M.; GONZÁLEZ GUITIAN, C. Buscar en Medline con PubMed. Coruña: Biblioteca del Complexo Hospitalario Universitario de A. Coruña, 2009. Disponível em: <http://bit.ly/2kV3Ozz>. Acesso em: 15 fev. 2017.

STROM, B. L. What is pharmacoepidemiology? In: STROM, B. L.; KIMMEL, S. E. (Ed.). Textbook of pharmacoepidemiology. Pennsylvania: John Wiley $\&$ Sons, 2006. p. 3-12.

TRIVIÑO, Z.; SANHUEZA, O. A. Paradigmas de investigación en enfermería. Ciencia y Enfermería, Concepción, v. 11, n. 1, p. 17-24, 2005. Disponível em: <http://bit.ly/1F4tWju>. Acesso em: 15 fev. 2017.

ZAVALA-GONZÁLEZ, M. A.; LIMA-ORTIZ, R.; GALLEGOS-AGUILAR, M. M. Utilización de hipoglucemiantes orales en una unidad médica familiar de Comalcalco, Tabasco, México, 2013. Revista Mexicana de Ciencias Farmacéuticas, México, DF, v. 45, n. 3, p. 81-85, 2014. Disponível em: <http://bit.ly/1ViyUxO>. Acesso em: 15 fev. 2017.

ZAVALA-GONZÁLEZ, M. A.; SÁNCHEZ-SANTANA, R. Calidad de prescripción de antibióticos en el servicio de pediatría de un Hospital General de Cárdenas, Tabasco, México, 2010. Revista Mexicana de Ciencias Farmacéuticas, México, DF, v. 45, n. 2, p. 37-42, 2014. Disponível em: <http:// bit.ly/2dTdSMn>. Acesso em: 15 fev. 2017.

\section{Contribución de los autores}

Zavala-González concibió y diseñó la investigación, recolectó y sistematizó la información, redactó el artículo y condujo el proceso de publicación. Todos los autores analizaron los datos y los interpretaron, revisaron críticamente el manuscrito y aprobaron tanto sus versiones preliminares como la versión final.

Recibido: 06/11/2017

Re-presentado: 21/05/2018

Aprobado: 02/07/2018 